\title{
Original
}

\section{Incidencia de esclerosis lateral amiotrófica en Costa Rica}

\author{
Pilar Rodríguez- Paniagua, Isaías Salas- Herrera, Mayra Cartín- Brenes
}

\section{Resumen}

\begin{abstract}
Justificación y objetivo: Hasta el momento no se ha publicado un estudio que nos permita conocer cuál es la incidencia de ELA en nuestro país. El objetivo de este estudio fue establecer la incidencia de esta enfermedad en Costa Rica, y describir las características de los pacientes diagnosticados en nuestro país desde enero de 1998 a noviembre de 2001.
\end{abstract}

Materiales y métodos: Utilizando diferentes fuentes de información se identificaron los casos de ELA en el periodo de 1998 al 2001. Se calculó la tasa de incidencia por persona año, se analizaron variables como sexo, distribución geográfica, grupo étnico, edad al diagnóstico, clasificación de la enfermedad, métodos diagnósticos y tratamientos.

Resultados: Entre enero de 1998 y diciembre del 2001, 102 casos de ELA son diagnosticados en Costa Rica, la tasa incidencia anual en población de 15 años y mas es de 0.97 (I.C. 95 \% 0.8 a 1.2) por 100.000 personas año. Se revisaron 76 expedientes. Veintiocho fueron mujeres y cuarenta y ocho hombres, presentándose una mayor incidencia en hombres, con una razón de masculinidad de 1.7, todos de raza blanca. La edad al diagnóstico fue de 58.4 años para las mujeres y 53.1 años para los hombres $(\mathrm{p}=0.15)$. El $75 \%$ de los casos se encuentran en tres provincias: San José, Alajuela y Cartago, con tasas de incidencia similares. La tasa de incidencia aumenta conforme aumenta la edad en las mujeres, mientras en los hombres se muestra la tasa más alta en la década de los sesenta. El 36.8\% de los casos fueron clasificados como ELA bulbar, mientras que el 55.3\% como espinal, el 7.9\% restante como bulbo espinal.

Conclusión: El estudio muestra cómo la incidencia de esta enfermedad es similar a la de los países que muestran las incidencias mas bajas. La incidencia se mantuvo estable en el período analizado, la enfermedad afecta más a hombres que a mujeres.

Descriptores: Esclerosis lateral amiotrófica, incidencia, Costa Rica

Key words: Amyotrophic Lateral Sclerosis, incidence, Costa Rica

Centro Nacional de Control del Dolor y Cuidados Paliativos, Caja Costarricense de Seguro Social

Abreviaturas: APELA, asociación para personas con esclerosis lateral amiotrófica; ELA, esclerosis lateral amiotrófica; FDA, food and drug administration; NMI, neurona motora inferior; NMS, neurona motora superior; SNC, sistema nervioso central Correspondencia Dirección electrónica: pilirodriguez@yahoo.com

ISSN 0001-6002/2007/49/1/31-35 Acta Médica Costarricense, @2007 Colegio de Médicos y Cirujanos
Recibido: 8 de julio de 2005

Aceptado: 7 de noviembre de 2006

La esclerosis lateral amiotrófica (ELA) es la enfermedad más común de la neurona motora, cuyo rasgo característica es la afección selectiva de las neuronas motoras del cerebro y médula espinal. Fue descrita inicialmente por Jean Martin Charcot en $1874 .{ }^{2}$ Es rápidamente progresiva, con una sobrevivencia media de dos a tres años después del diagnóstico. ${ }^{3}$

La incidencia en varios países como Italia, Irlanda, Escocia y Estados Unidos oscila entre 0.6 y 2.8 por 100.000 habitantes de 15 años y mas por año. ${ }^{4-6}$ Con una prevalencia de 1.6 a 8.5 por 100.000 habitantes. $^{6}$

Hasta el momento su causa permanece desconocida, aunque se han postulado varias teorías. Entre ellas, factores genéticos, intoxicación por metales pesados, deficiencia de calcio y magnesio, factores tóxicos en la dieta, tabaquismo, factores inmunológicos, trauma, exposición a campos electromagnéticos e infecciones virales. . $^{1,-9}$ 
El diagnóstico es clínico, basado en las manifestaciones característicasdedebilidadprogresiva, atrofia, fasciculaciones e hiperreflexia que afectan diferentes áreas corporales. ${ }^{10} \mathrm{En}$ 1991, la Federación Mundial de Neurología estableció criterios diagnósticos para fines de investigación (El Escorial) que fueron revisados en 1997 (Airlie House) para asegurar la uniformidad dentro de los ensayos clínicos. ${ }^{11}$

El cuadro clínico puede variar dependiendo de la localización y progresión de los cambios patológicos. De esta manera la ELA se puede clasificar como bulbar, espinal o generalizada, de acuerdo a las manifestaciones clínicas que se presenten al momento del diagnóstico. En la ELA bulbar se presentan con más frecuencia trastornos del lenguaje, como ronquera, y disatria, disfagia y disnea como síntomas iniciales. Mientras que la espinal se presenta como debilidad progresiva que predispone a los pacientes a caídas, dificultad para vestirse y desarrollar sus actividades de la vida diaria. ${ }^{10,11}$

ELA continúa siendo una enfermedad fatal. Se han hecho avances en la última década, pero aún no se cuenta con una terapia efectiva. ${ }^{2}$ El Riluzole es la única droga aprobada por la FDA para ser utilizada en estos pacientes. ${ }^{10}$ El tratamiento continúa siendo paliativo. Sin embargo, es mucho lo que se puede hacer desde el punto de vista integral por estos pacientes y sus familiares. La meta en estos pacientes es lograr un adecuado manejo de los síntomas que se puedan presentar para mejorar su calidad de vida. ${ }^{12}$

El objetivo de este estudio fue establecer la incidencia de ELA en Costa Rica y describir las características de la población total de pacientes diagnosticados en nuestro país de enero de 1998 a noviembre de 2001.

\section{Materiales y métodos}

Este es un estudio descriptivo retrospectivo. Los pacientes que participaron en esta investigación fueron diagnosticados por los médicos de los Servicios de Neurología de los diferentes hospitales nacionales, durante el período de 1998 al 2001. Fueron identificados de varias maneras: en primera instancia, los Servicios de Bioestadística nos entregaron un listado con los pacientes que egresaron de los diferentes hospitales con el diagnóstico de ELA. Posteriormente, dado que el Riluzole es de uso exclusivo para estos pacientes, se solicitó la ayuda de los Departamentos de Farmacia de todos los hospitales del país, quienes brindaron un informe del registro de pacientes que han utilizado esta droga en los años determinados. Así mismo, se contactó al Servicio de Bioestadística de la Caja Costarricense de Seguro Social con el fin de obtener el reporte anual nacional de esta enfermedad. Finalmente la Asociación de Personas con Esclerosis Lateral Amiotrófica "Lic. Víctor Hugo Núñez Torres” (APELA), suministró un listado con los números telefónicos de sus miembros y se procedió a contactarlos telefónicamente con la intención de obtener los datos epidemiológicos para el estudio.

El Servicio de Bioestadística reportó que en 1998 se presentaron 24 casos, en 199925 casos y en el año 200027 casos, haciendo una proyección para el año 2001, se estimó que se presentarán aproximadamente 26 casos, dando un total estimado de 102 casos en el período en estudio.

Se analizaron variables como sexo, distribución geográfica, grupo étnico, edad promedio de diagnóstico, clasificación de la enfermedad, métodos diagnósticos y tratamientos.

Para la clasificación de la enfermedad en espinal o bulbar, se respetó el criterio de los neurólogos que hicieron el diagnóstico.

Aspectos éticos. El protocolo de este estudio fue revisado y aprobado por el Comité de Ética e Investigación del Hospital Dr. Rafael Ángel Calderón Guardia.

Análisis de los datos. Se obtuvo la tasa de incidencia por persona año, los intervalos al 95\% de confianza se calcularon asumiendo el comportamiento de una variable que sigue la distribución de Poisson. ${ }^{13}$ Se realizó distribución de frecuencias de todas las variables; para las variables cualitativas se analizaron los porcentajes de las categorías identificadas. Para las variables cuantitativas se describieron las medidas de tendencia central, de dispersión y de posición.

Se realizó análisis bivariado crudo para valorar posibles asociaciones entre variables nominales; y comparación de promedios para las variables cuantitativas. Los resultados obtenidos se sometieron a pruebas de significancia estadística: para las variables nominales se aplicó el ChiCuadrado, bajo la Hipótesis nula de de Independencia. Para las métricas la prueba t de Student. Se consideró significativo cuando la $\mathrm{p}<0,05$.

Las tasas de incidencia se calcularon utilizando las proyecciones poblacionales para los años 1998-2001 del Instituto Nacional de Estadística y Censos, tomándose en cuenta a los mayores de 15 años, y separándose por sexo, edad y provincia de residencia.

Los datos fueron analizados en EPI-INFO 6.4 del Centro para el Control de Enfermedades (CDC) de Atlanta, Estados Unidos.

\section{Resultados}

Se calculó la incidencia anual y para el período en estudio, como se muestra en el Cuadro 3, la tasa se mantiene muy similar. 
Utilizando las distintas fuentes de información mencionadas fue posible revisar un total de 76 historias clínicas: 20 casos en 1998, 12 casos en 1999, 18 casos en 2000 y 26 casos en 2001. Veintiocho fueron mujeres y 48 hombres, presentándose una razón de masculinidad de 1.7, todos de raza blanca. La edad promedio de diagnóstico fue de 54.4 años para las mujeres, con un rango entre $18.73 \mathrm{y}$ 87.16 años. Para los hombres fue de 53.1 años, con un rango entre 23.41 y 77.32 años. No existen diferencias significativas entre sexos $(p=0.15)$. La incidencia aumenta conforme aumenta la edad en las mujeres, mientras en los hombres las tasas mas altas se muestran en la década de los sesenta, como puede apreciarse en la Figura 1.

La distribución geográfica se agrupó por provincias, el 75\% de los pacientes residen en tres provincias: San José (44.15\%), Alajuela (19.48\%) y Cartago (11.68\%), mostrando tasas de incidencia muy similares para el período en estudio, como se presenta en el Cuadro 2.
El 36.8\% de los casos fueron clasificados como ELA bulbar, mientras que el 55.3\% como espinal, el 7.9\% restante como bulbo espinal.

De los 76 casos el 75\% toman Riluzole como parte de su tratamiento. El 19.7\% utilizan sonda de alimentación por gastrostomía, y solamente el 6.6\% están actualmente con ventilación mecánica asistida.

Cabe destacar que un $21.1 \%$ de los casos no cuenta con estudios electrofisiológicos como parte de su protocolo de diagnóstico.

No se encontró asociación entre el consumo de Riluzole y el sexo $(p=0.582)$. Tampoco en el uso de ventilación mecánica asistida y el sexo, con $(\mathrm{p}=0.879)$. Así como tampoco en el uso de gastrostomía y el sexo $(p=0.777)$. De la misma forma, no se observó asociación entre el tipo de la enfermedad y el sexo $(p=0.419)$.

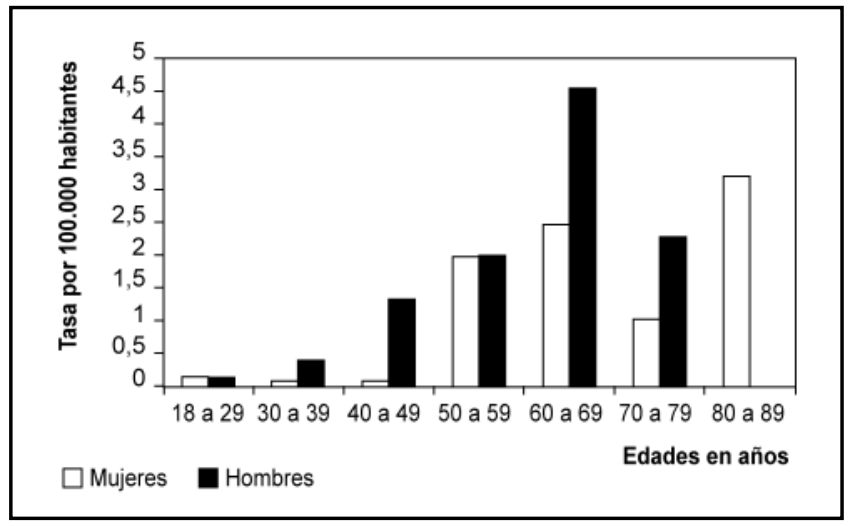

Figura 1. Tasas incidencia ELA según edad, Costa Rica 1998-2001.

\begin{tabular}{|ccc|}
\hline $\begin{array}{c}\text { Cuadro 2. Tasa de incidencia de Esclerosis } \\
\text { Lateral Amiotrófica, según edad } \\
\text { Costa Rica 1998 - 2001 }\end{array}$ \\
\hline \multicolumn{3}{|c|}{ Combres } \\
\hline Grupos edad & Mujeres & Hombr| \\
\hline 18 a 29 & 0.15 & 0.14 \\
30 a 39 & 0.08 & 0.40 \\
40 a 49 & 0.12 & 1.34 \\
50 a 59 & 1.99 & 2.00 \\
60 a 69 & 2.48 & 4.55 \\
70 a 79 & 1.03 & 2.29 \\
80 a 89 & 3.20 & 0.00 \\
\hline Total & 0.54 & 0.90 \\
\hline
\end{tabular}

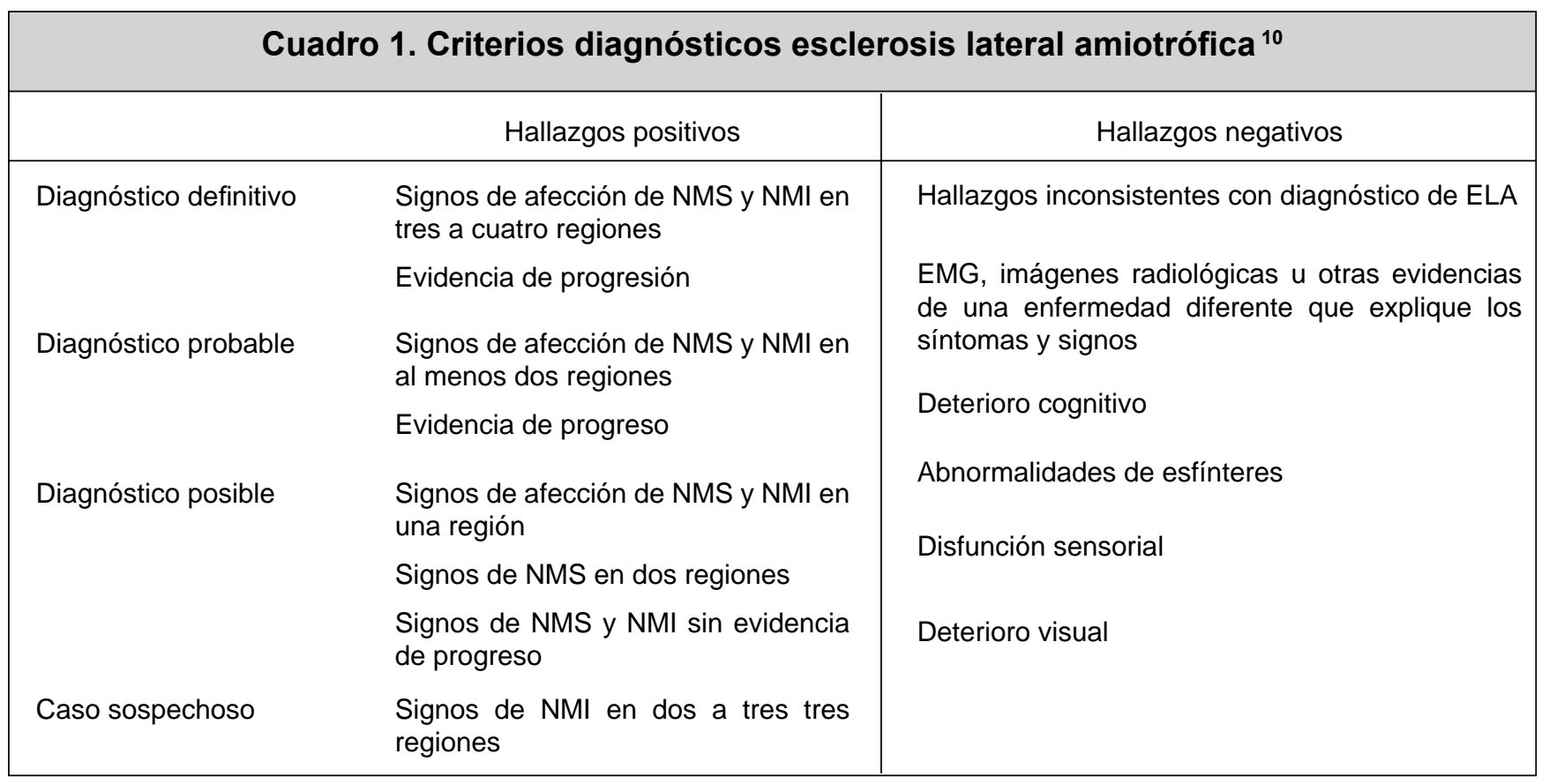




\begin{tabular}{|c|c|c|c|c|c|}
\hline \multicolumn{6}{|c|}{$\begin{array}{l}\text { Cuadro 3. Tasa de incidencia anual Esclerosis } \\
\text { Lateral Amiotrófica en Costa Rica, de } 1998-2001\end{array}$} \\
\hline \multirow{2}{*}{$\begin{array}{l}\text { Año } \\
1998\end{array}$} & \multirow{2}{*}{$\begin{array}{c}\text { casos } \\
24\end{array}$} & \multirow{2}{*}{$\begin{array}{l}\text { población } \\
\geq 15 \text { años } \\
2508003\end{array}$} & \multirow{2}{*}{$\begin{array}{c}\text { tasa } \\
/ 100.000 \\
0.96\end{array}$} & \multicolumn{2}{|c|}{ I.C. $95 \%$} \\
\hline & & & & 0.6 & 1.4 \\
\hline 1999 & 25 & 2591288 & 0.96 & 0.6 & 1.4 \\
\hline 2000 & 27 & 2678058 & 1.01 & 0.7 & 1.5 \\
\hline 2001 & 26 & 2765266 & 0.94 & 0.6 & 1.4 \\
\hline Total & 102 & 10542615 & 0.97 & 0.8 & 1.2 \\
\hline
\end{tabular}

La edad del diagnóstico y el sexo tampoco mostró diferencias $(\mathrm{p}=0.1455)$

\section{Discusión}

La Esclerosis Lateral Amiotrófica a pesar de ser una enfermedad poco conocida en nuestro medio, no es una enfermedad infrecuente. Sin embargo, este es el primer estudio que se realiza a nivel nacional acerca de su incidencia. Las características retrospectivas del estudio abren la puerta a la subestimación del número real de casos. Se hará un nuevo estudio de tipo prospectivo, que permita acercarnos más a esta enfermedad en Costa Rica. Los estudios prospectivos se reconocen por ser más útiles que los estudios retrospectivos para cuantificar la incidencia de enfermedades poco frecuentes como esta.

La incidencia de esta enfermedad en nuestro país es similar a la de los países que muestran las incidencias más bajas, lo que hace pensar que existe un subregistro.

Esta enfermedad afecta más a los hombres que a las mujeres, y su incidencia aumenta conforme la edad. Como se reporta en otros estudios se presentó un incremento rápido después de los 40 años $^{3,5}$, con un pico entre los 50 y los 70 años, y una disminución en la población de 70 y más años. Llama la atención que en este último grupo poblacional se presentó un mayor número de casos del sexo femenino.

Es muy importante resaltar que un alto número de estos pacientes consume Riluzole, el cual, aunque en poco, aumenta la supervivencia de los mismos. Además, desempeña un papel fundamental en el manejo del concepto de la incurabilidad de esta enfermedad para los familiares y pacientes. A pesar de esto, son pocos los pacientes que optan por medidas más agresivas para paliar sus síntomas. Esto concuerda con el estudio de Albert et $\mathrm{al}^{14}$, el cual reporta que solamente el $5.6 \%$ de los pacientes apoyan el uso de la ventilación mecánica asistida; mientras que el 28.2\% están de acuerdo con la colocación de la sonda para alimentación por gastrostomía.

\begin{tabular}{|lccccc|}
\hline \multicolumn{5}{|c|}{$\begin{array}{c}\text { Cuadro 4. Tasa de incidencia de Esclerosis } \\
\text { Lateral Amiotrófica, por provincia, Costa Rica } \\
1998-\mathbf{2 0 0 1}\end{array}$} \\
\hline Año & casos & $\begin{array}{c}\text { población } \\
\geq 15 \text { años }\end{array}$ & $\begin{array}{c}\text { tasa } \\
\text { I100.000 }\end{array}$ & I.C.95\% \\
\hline San José & 34 & 3852673 & 0.87 & 0.6 & 1.2 \\
Alajuela & 15 & 1955738 & 0.76 & 0.4 & 1.2 \\
Cartago & 9 & 1191703 & 0.74 & 0.3 & 1.4 \\
\hline
\end{tabular}

Por otro lado, al $78.9 \%$ de los casos no se le realizaron estudios electrofisiológicos. Esto recuerda que el diagnóstico de la enfermedad continúa siendo clínico. Sin embargo, consideramos que este tipo de estudios clínicos son de gran importancia para aclarar el diagnóstico.

Por último, cabe destacar que, para continuar realizando otros estudios que permitan conocer más acerca de la ELA y su comportamiento en Costa Rica, es necesario unificar los criterios para su diagnóstico. De esta manera se proporciona una forma más estandarizada de analizar y comparar los datos. La clasificación de El Escorial, con su última modificación en 1997, y aceptada por la Federación Mundial de Neurología, debe ser la pauta por seguir para diagnosticar a estos pacientes.

\section{Abstract}

Objective. Amyotrophic lateral sclerosis is a degenerative disease of the central nervous system that is characterized by progressive affection of the upper and lower motor neuron. Until now, there has not been a publication that refers to its incidence in our country. The present investigation is based on the analysis of patients seen with the diagnosis of this illness from January 1998 to November 2001.

Methods. Incidence rate per person-years was calculated and variables such as sex, age of diagnosis, ethnics, geographic distribution, classification of the disease, treatment and diagnostic methods were analyzed.

Results. Between January 1998 and December 2001 a total of 102 cases were diagnosed with ALS in Costa Rica, the annual incidence rate 0.97 (I.C. $95 \% 0.8$ a 1.2) per 100.000 person-years. 76 medical records were reviewed. Twenty eight were females and forty eight males, the M: F ratio was 1.7. The average age of diagnosis was 58.4 years for females and 53.1 years for males $(p=0.15)$. The geographic distribution showed that $75 \%$ of the patients live in San José, Alajuela and Cartago, with a very similar incidence rate. The incidence increases with age in women, while in men shows a peek of onset at the sixth decade. 
Conclusion. The study shows that the incidence of ALS in our country appears to be similar to those countries with lower rates of incidence. The incidence rate was stable in the years of the study; the disease affects more men than women.

\section{Referencias}

1. Nelson L, McGuire V, Longtreth, Matkin C. Population-Based Casecontrol study of amyotrophic lateral sclerosis in western Washington State. I Cigarette smoking and alcohol consumption. Am J Epidemiol 2000; 151:156-163

2. Rowland L, Shneider N. Amyotrophic Lateral Sclerosis. N Engl J Med 2001; 344:1688-1699.

3. Nelson L, McGuire V, Longtreth, Matkin C. Population-Based CaseControl Study of Amyotrophic Lateral Sclerosis in Western Washington State. II Diet. Am J Epidemiol 164-172

4. Chió A. Incidence of ALS in Italy. Neurology 2001; 56: 239-244.

5. McGuire V, Longstreth W T, Koepsell T, van Belle G. Incidence of Amyotrophic Lateral Sclerosis in three counties in western Washington State. Neurology 1996; 47:571-573.
6. Traynor BJ, Codd MB, Corr B, Forde C, Frost E, Hrdiman O. Incidence and prevalence of ALS in Ireland. 1995-1997. A population-based study. Neurology 1999; 52:504-509.

7. Brooks B. Clinical Epidemiology of Amyotrophic Lateral Sclerosis. Neurol Clin 1996; 54:399-409.

8. Johansen C, Olsen J. Mortality from Amyotrophic lateral Sclerosis, Other Chronic Diseases, and Electric Shocks among Utility Workers. Am J Epidemiol 1998;148:362-368.

9. Riggs J. Amyotrophic Lateral Sclerosis, Heterogeneous Susceptibility, Trauma, and Epidemiology. Arch of Neurol 1996;53:225-227.

10. Walling A. Amyotrophic lateral Sclerosis: Lou Gehrig's disease. American Family Physician 1999;59:1489-1495.

11. Traynor B, Codd M, Corr B, Forde C, Frost E, Hardiman O. Clinical features according to El Escorial and Airlie House diagnostic criteria. Arch Neurol 2000;57: 1171-1176.

12. Miller R.G., Rosenberg J.A., Gelinas D.F., Mitsumoto H, Newman D, Sufit $\mathrm{R}$ et al. Practice parameter: The care ot the patient with amyotrophic lateral sclerosis (an evidence based review). Neurology 1999;52:1311-1321.

13. Szklo M, Nieto J. Epidemiology: Beyond the Basics. Aspen Publishers, Inc, 2000

14. Albert S M, Murphy P L, Del Bene M L, Rowland L P. A prospective study of preferences and actual treatment choices in ALS. Neurology 1999;53:278-283. 